September 21-25, 2020, Virtual, Online

\title{
VORTEX LATTICE METHOD FOR THE CALCULATION OF THE TIP LEAKAGE FLOW: EVALUATION ON A SINGLE BLADE
}

\author{
Christophe Montsarrat \\ Fluid Mechanics and \\ Acoustics Laboratory (LMFA) \\ Turbomachinery Department \\ Ecole Centrale de Lyon \\ Ecully, France \\ Mail: christophe.montsarrat@ec-lyon.fr
}

\author{
Benjamin Deveaux \\ ONERA - the French Aerospace Lab \\ Meudon, France \\ Mail: benjamin.deveaux@onera.fr
}

\author{
Jérôme Boudet \\ Fluid Mechanics and \\ Acoustics Laboratory (LMFA) \\ Turbomachinery Department \\ Ecole Centrale de Lyon \\ Ecully, France \\ Mail: jerome.boudet@ec-lyon.fr
}

\author{
Julien Marty \\ ONERA - the French Aerospace Lab \\ Meudon, France \\ Mail: julien.marty@onera.fr
}

Eric Lippinois

Safran Aircraft Engines

Moissy-Cramayel, France

Mail: eric.lippinois@safrangroup.com

\section{ABSTRACT}

This paper investigates the use of a Vortex Lattice Method to simulate tip-leakage flow with small computational effort. The module PyLiSuite presented in the present paper has been completely developed from scratch and is validated on twodimensional and three-dimensional basic cases. The validation lies on the estimate of the lift coefficient computed from the circulation given by the module. The capabilities of PyLiSuite regarding tip-leakage flow are gauged in comparison with novel experimental measurements on a single blade with an adjustable gap. The results show a good prediction of the shape and size of the tip-leakage vortex for large tip gaps. Differences in the position and the deficit of static pressure in the core of the vortex are noted. The future improvements on the module concern the influence of viscosity to be accounted for and the computation time which could be shortened.

\section{NOMENCLATURE}

$\alpha \quad$ Angle of attack

$\beta \quad$ Relative flow angle

$\eta \quad$ Angle coordinate used for the flat plate configuration

$\Gamma_{\text {blade }}$ Circulation of the blade

$\Gamma_{T L V} \quad$ Tip leakage vortex circulation

$\Gamma_{2 d} \quad$ Part of the TLV circulation at constant radius

$\Gamma_{i} \quad$ Induced circulation related to shed vorticity

$\Gamma_{r e f} \quad$ Equivalent constant work design circulation

$\tau \quad$ Tip gap size

$b \quad$ Span

$c \quad$ Chord length

$C_{L} \quad$ Lift coefficient

$c_{p} \quad$ Pressure coefficient

$P_{S} \quad$ Static pressure

$P_{\infty} \quad$ Freestream static pressure

$r_{\text {tip }} \quad$ Radius at the tip

$r_{h u b} \quad$ Radius at the hub 


$\begin{array}{ll}S & \text { Surface area of reference } \\ U_{\infty} & \text { Freestream velocity } \\ V & \text { Absolute flow velocity } \\ V_{x} & \text { Axial velocity } \\ W & \text { Relative flow velocity } \\ \text { AR } & \text { Aspect ratio } \\ \text { TLF } & \text { Tip-leakage flow } \\ \text { TLV } & \text { Tip-leakage Vortex } \\ \text { TE } & \text { Trailing Edge } \\ \text { VLM } & \text { Vortex Lattice Method } \\ \text { PyLiSuite } & \text { VLM module developed and presented }\end{array}$

\section{INTRODUCTION}

Secondary flows represent a significant limitation for the design of compressors in turbomachines. The flow observed in the clearance between the casing and the tip of rotor blades due to the pressure difference between the two sides of the blade is responsible for the generation of vorticity and important losses. Tip-leakage flows (TLF) can represent up to one third of the losses [3] of a compressor and their influence in the onset of instabilities make them a physical phenomenon of big interest in turbomachinery. The formation of the tip-leakage vortex (TLV) itself and its interaction with the rest of the flow (boundary layer, shock, other sources of vorticity) still need to be better understood. High-resolution simulations such as Large Eddy Simulations (LES) allow the turbulent structures of the vortex to be well depicted and the interaction between structures of different scales of energy is then possible to be captured. These methods are increasingly popular thanks to the continuous progress with CPU. They are quite useful tools to give a more solid physical comprehension, especially regarding secondary flows, but they are still very costly and parametric studies remain complicated to understand the sensitivities playing on TLF. The purpose of this paper is precisely to investigate TLF with a numerical lowcost method, dedicated to physical analysis and large parametric studies.

\subsection{Tip-leakage flow}

Rains was one of the first to study tip-leakage flow [20]. He proposed a very simple model estimating the mass flow rate of the leakage flow as a function of the pressure difference across the blade. He divided the flow into two main regions: a first region of acceleration of the fluid where a vena contracta forms and a second region where mixing takes place with the mainstream flow and generates losses. You et al. performed LES simulations on cascades to have a better view of the topology of TLF [24]. They identified three types of vortices in the clearance: the TLV, most energetic, the tip-separation vortex, and one or multiple induced vortices formed by the interaction of the TLV with the endwall boundary layer [2].

Three strategies to model the tip-leakage flow have been identified in the literature. The first approach consists of dealing with the mixing region. It has been done by Storer and Cumpsty [21], as well as Denton [3]. They built similar models regarding the losses in that region by considering the increase in entropy due to the difference in direction between the leakage and the mainstream flows. The second approach considers the TLV itself and the losses generated by the vorticity associated $[13,16]$. The method presented in the present paper could be classified as such. Another way of modelling TLF is to estimate the increase in the blockage area induced by the presence of TLV. Khalid et al. [9] modelled the growth of the blockage area of different configurations representative of compressors. Their works also showed the influence of TLF in the onset of instabilities such as rotating stall, following the works of Koch and Smith [10,11]. Vo et al. [23] and Hoying et al. [6] proposed an explanation for the phenomenon observed before the outbreak of rotating stall or even surge: two conditions must be satisfied and are both related to TLF. These instabilities significantly reduce the operating range of compressors and impose safety margins to ensure that the off-design conditions remain stable.

\subsection{Vortex Lattice Method}

Prandtl's lifting line theory [19] has been widely used on aircrafts and propellers. This theory mimics the presence of an aerodynamic object by a distribution of circulation $\Gamma(y)$ spanwise. An important assumption is made on the aspect ratio of the object with this theory, which must be much greater than 1 . The purpose of the lifting line is to calculate the distribution of circulation bound to that object and estimate the vorticity generated and shed by it. This approach is a good way of estimating the induced drag generated by very large aspect ratio objects such as airplane wings. The idea is that the vorticity shed downstream of the line modifies the local incidence and deviates the aerodynamic force on the object, converting part of the lift into induced drag. Lashminarayana and Horlock [13] applied this approach on TLF. The tip gap configuration is modelled by a slotted wing with an adjustable midspan gap, half the gap replicating the tip gap with the condition of symmetry representing the casing. They studied the influence of the size of the gap on the trajectory and the strength of the TLV. For tip gap-to-chord ratios $\lambda>1$, their model based on the lifting line theory yields good predictions regarding the calculation of the circulation associated to the tipleakage vortex (TLV) but the method does not fit for gaps with a dimension lower than the chord. Their explanation lies in the fact that viscous effects are no longer negligible. The drag coefficient estimated with the method is overestimated compared to their experiment on the slotted wing. In their model, they introduce a coefficient $K$ accounting for these viscous effects for what 
they call the retained vorticity, which is therefore not shed in the tip leakage vortex. The resulting circulation for small $\lambda$ equals $(1-K) \Gamma_{2 d}$, where $\Gamma_{2 d}$ corresponds to an uniform circulation around the blade, corresponding to a case for which no vorticity is shed. The coefficient $K$ was determined empirically from their measurements [14].

Based on this work, it is possible to calculate the decrease in efficiency from the sum of the losses due to induced drag associated to the TLV, and the losses due to the blade boundary layers [12]. A more realistic approach yielding the blade-to-blade flow characteristics is also developed in the article, on a cascade configuration. An analytical vortex model is used with three parameters whose evolutions along the axis can be estimated: the vortex core radius, the pitchwise position of the vortex in the passage of the cascade and the vorticity. The local estimate of these parameters leads to a better prediction of the deviation and the losses. This second model is of great interest since it offers a $(r, \theta)$ map of the flow characteristics in a blade passage.

The inherent problem of this approach is its high dependence on correlations calibrated on experimental measurements. The validity and generality of the models is therefore difficult to establish. For this model, the coefficient $K$ introduced for low tip-to-chord ratios is particularly complicated to estimate theoretically without resorting to correlations. The problem is in fact double with the lifting line approach for compressor rows. First, the order of magnitude of the aspect ratio for compressor blades is about 1 , which contradicts the assumption made at first. Second, the tip gap introduces a length scale smaller than chord length, which also contradicts the description of the blade as a spanwise line.

To go beyond the limitations of the assumptions made in Prandtl's lifting line theory (mostly large aspect ratios), a solution is to extend it to two dimensions by considering a surface discretized into panels of vorticity. This approach is called Vortex Lattice Method (VLM) [8, 15]. This introduces a chordwise discretization for a more realistic description of the tip gap flow. To our knowledge, few studies have been done on tip-leakage flow using this kind of approach $[17,18]$. This paper is intended to validate the code developed based on VLM and gauge the possibilities of this method regarding TLF, by comparing the numerical results to experimental measurements on a single blade configuration. The method is here applied to incompressible flows, conditions satisfied in the experiment used for the comparisons. The main idea is to be able to avoid as much as possible to use correlations, which are hardly applicable to innovative compressor configurations.

\section{NUMERICAL METHOD}

\subsection{Calculation of the circulation}

The Vortex Lattice Method (VLM), here introduced, adds one dimension, chordwise, to the problem to be solved. The

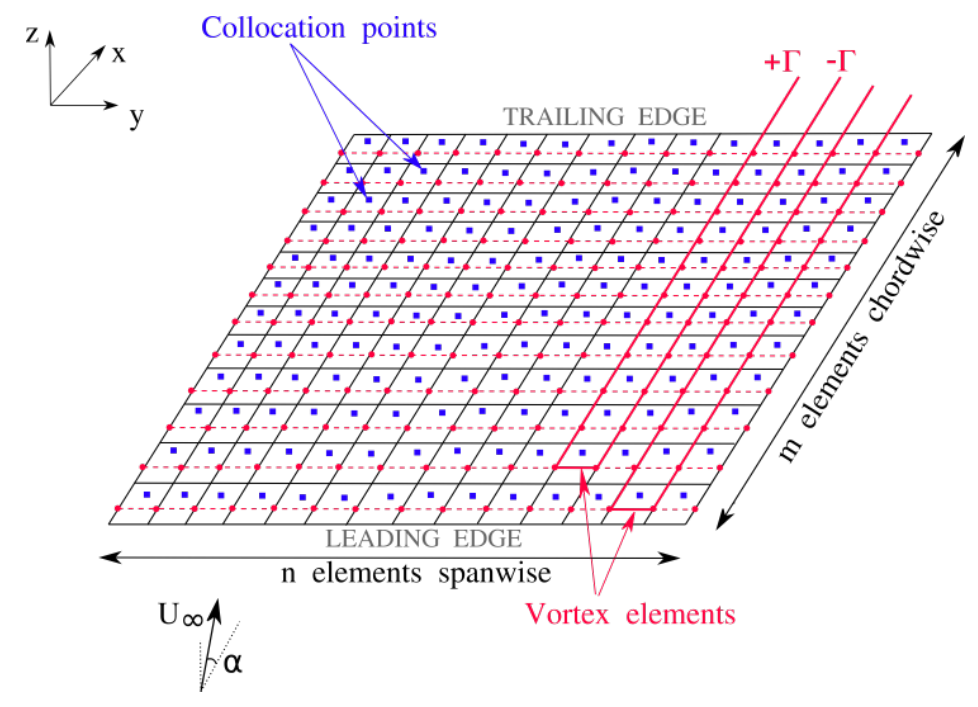

FIGURE 1: SCHEMATIC OF THE VORTEX LATTICE METHOD FOR A FLAT PLATE SUBMITTED TO A FLOW WITH AN ANGLE OF ATTACK $\alpha$. THE FLAT PLATE IS HERE DISCRETIZED INTO $n \times m$ VORTEX PANELS.

principle of the approach is the same but instead of discretizing a spanwise line, the surface considered is divided into panels to which is attached a circulation. Figure 1 shows the discretization of a flat plate and the main parameters of the flow. A horseshoe vortex is attached to each panel. It consists of one branch bound to the surface (the head) and two branches extending downstream as straight lines with circulations $\pm \Gamma$ (the legs), forming the wake, here prescribed in the axial direction $x$. The different bound circulations can be calculated by solving the linear system associated to this problem by imposing a slip condition on the lifting surface $\vec{V} \cdot \vec{n}=0$.

Vortex and collocation points are placed upon each panel of the surface grid at very specific positions. Vortex points are located at $1 / 4$ of the width of the panel, in the axial direction, and control points, where the slip condition applies, are placed at $3 / 4$ of the same length on each panel, at midspan of each vortex panel. The position of the bound vortices is crucial since it determines where the singularities are situated:

1. The Kutta condition which ensures that the circulation at the trailing edge is zero,

2. The square root singularity at the leading edge.

James et al. [7] investigated the impact of the position of these points, yielding the same conclusion. Positioning the points elsewhere on the grid changes the singularities and leads to an inaccurate definition of the problem to be solved. Significant errors are made and even non-physical values of the solutions are obtained. The matrix of the linear problem to be solved may even become singular with singularities badly located. 
Curved surfaces can be discretized with panels as shown in section 3.3.1. The calculation of the lift coefficient is done using Kutta-Jukowski's theorem once the unknown circulations attached to the surface have been computed. The circulation attached to each panel contributes to the total circulation of the lifting surface and consequently to the lift coefficient:

$$
C_{L}=2 \frac{\Gamma}{U_{\infty} S}
$$

where $\Gamma$ is the sum of the circulations of the panels and $S$ is the wet area on which the panels are distributed.

The method is also able to calculate the velocity components associated to the circulation of the panels anywhere in the field, using Biot-Savart's law.

\subsection{Application to turbomachinery}

Considering a rotor row in the relative frame of reference, the radial distribution of blade loading can be calculated from the distribution of blade circulation [5]:

$$
\Delta h_{t}=-\frac{r \omega \Gamma_{\text {blade }}}{s}=-\frac{\omega N_{\text {blades }} \Gamma_{\text {blade }}}{2 \pi}
$$

where $\omega$ is the rotational speed of the row and $s$ the pitch of the row, showing that the blade loading distribution is directly proportional to the blade circulation.

Euler's relation for turbomachinery yields, in the rotor frame of reference

$$
\Delta h_{t}=\Delta\left(r \omega W_{\theta}\right)=r \omega \Delta W_{\theta}
$$
blade:

Indeed, the blade circulation can be calculated around the

$$
\Gamma_{\text {blade }}=-s \Delta W_{\theta}=-s\left(V_{x_{2}} \tan \beta_{2}-V_{x_{1}} \tan \beta_{1}\right)
$$

The circulation can be decomposed into a 2D component, constant, hence shedding no vorticity, minus the shed vorticity circulation $\Gamma_{i}$ contributing to the TLV.

$$
\Gamma_{\text {blade }}=\Gamma_{2 d}-\Gamma_{i}
$$

$\Gamma_{\text {blade }}$ and $\Gamma_{i}$ can be calculated from which $\Gamma_{2 d}$ can be determined. This circulation is a constant from Helmholtz's theorems and therefore presents no variation spanwise, which corresponds

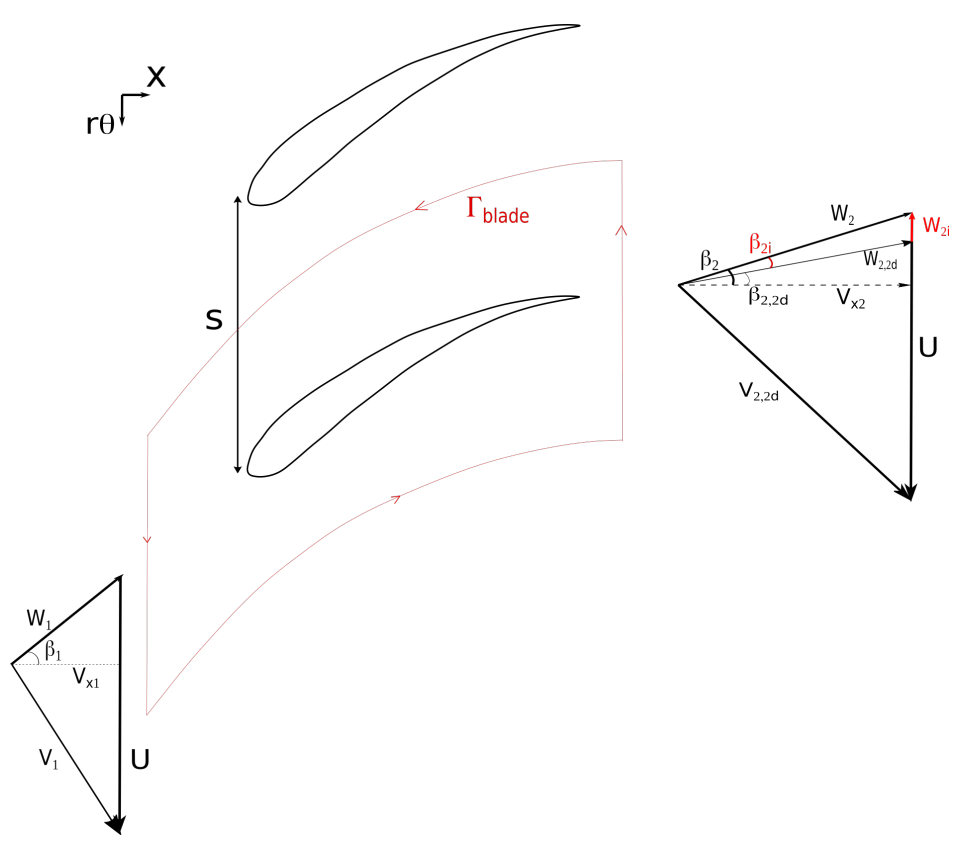

FIGURE 2: VELOCITY TRIANGLES AT A CONSTANT RADIUS ILLUSTRATING THE MODIFICATION OF THE DEVIATION $\beta_{2,2 d}$, HERE AN UNDERTURN, DUE TO THE PRESENCE OF TLV.

to no shed vorticity. Finally, using (4) and (5), and neglecting the influence of shed vorticity on $\beta_{1}$ and $V_{x}$, we can define a 2D outflow angle $\beta_{2,2 d}$ and an induced contribution $\beta_{i}$ with

$$
\Gamma_{2 d}=-s\left(V_{x 2} \tan \beta_{2,2 d}-V_{x 1} \tan \beta_{1}\right)
$$

$$
\Gamma_{i}=-s V_{x 2}\left[\tan \beta_{2,2 d}-\tan \left(\beta_{2,2 d}-\beta_{i}\right)\right]
$$

Given the distribution of circulation, it is therefore possible to retrieve the velocity triangles due to that circulation, here assuming a constant flow coefficient $V_{x 2} / U$ in the schematic. In reality, as well as the blade loading, the flow coefficient varies due to the presence of the induced circulation: their variation can be computed with Biot-Savart's law, yielding the velocity components.

From the design point of view, if one is only interested in reducing tip-leakage flows, a definition of the ideal case is a constant work design. In that specific case, the circulation $\Gamma$ does not depend on the radius of the blade since the loading is kept constant spanwise. Without any variation spanwise, no vorticity is shed downstream and the 3D effects do not impact the 2D 
design of the blade. A criterion to estimate the efficiency of the design regarding tip-leakage flows could be defined as the mean deviation between an equivalent constant work design with the mean circulation $\Gamma_{\text {ref }}$ and the actual design, i.e.

$$
D_{\text {design }}=\frac{1}{r_{t i p}-r_{h u b}} \int_{r_{h u b}}^{r_{\text {tip }}}\left|\frac{\Gamma_{\text {blade }}-\Gamma_{\text {ref }}}{\Gamma_{\text {ref }}}\right| \mathrm{d} r
$$

with

$$
\Gamma_{r e f}=\frac{1}{r_{t i p}-r_{h u b}} \int_{r_{h u b}}^{r_{t i p}} \Gamma_{\text {blade }}(r) \mathrm{d} r
$$

Defining a coherent and relevant measure of loss is challenging. The present method is inviscid and incompressible so there is no generation of entropy. Losses arise through induced drag and can be evaluated from shed vorticity.

\section{RESULTS}

The VLM solver PyLiSuite has been entirely coded from scratch and the results presented here aim at validating it. The validation can be separated into two parts:

1. The validation first deals with two dimensional cases by considering surfaces with very high aspect ratios

2. Three-dimensional validations are then considered on a parabolic wing against analytical results where the effect of the aspect ratio is investigated.

The capabilities of the code applied to a tip-leakage flow configuration are evaluated and compared to experimental measurements performed on a single blade for a variable tip gap size.

\subsection{D validation}

The flat plate The first case considered is the flat plate for which the analytical solution to the potential flow equations can be calculated. The validation is based on the prediction of the lift coefficient $C_{L}$ as a function of the angle of attack, $\alpha$ and on the distribution of the pressure coefficients $c_{p}$. The configuration is sketched in Fig. 3.

The theoretical lift coefficient $C_{L}=2 \pi \alpha$, predicted by Thin Airfoil Theory [1], is compared to the one calculated with VLM as the sum of the bound circulations of the panels, using KuttaJukowski's theorem.

PyLiSuite simulates a flat plate with an aspect ratio $A R=$ $b / c=1000$. The meshing uses a biharmonic distribution spanwise and chordwise to better capture the flow gradients, more significant near the leading edge and the trailing edge. The number of points in the two directions has been increased until a convergence on the lift coefficient has been achieved as summarized in

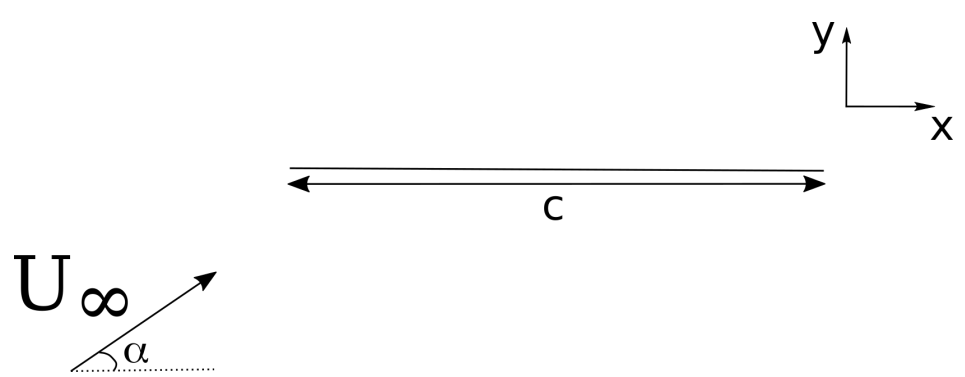

FIGURE 3: FLAT PLATE CONFIGURATION

\begin{tabular}{c|c|c} 
Grid size & $\frac{C_{L}}{2 \pi \alpha}$ with grid type 1 & $\frac{C_{L}}{2 \pi \alpha}$ with grid type 2 \\
\hline $10 \times 10$ & 0.997 & 0.976 \\
$20 \times 20$ & 0.996 & 0.990 \\
$30 \times 30$ & 0.996 & 0.993 \\
$40 \times 40$ & 0.996 & 0.994
\end{tabular}

TABLE 1: CONVERGENCE OF THE LIFT COEFFICIENT OBTAINED WITH PyLiSuite FOR THE FLAT PLATE WITH $A R=1000$ AND $\alpha=3^{\circ}$. THE GRID SIZE GIVES THE NUMBER OF POINTS CHORDWISE $\times$ SPANWISE FOR TWO TYPES OF GRIDS. BOTH USE THE SAME SPANWISE DISTRIBUTION. GRID 1 USES A BIHARMONIC DISTRIBUTION CHORDWISE, GRID 2 A REGULAR GRID. THE RELATIVE DIFFERENCE OBTAINED WITH THE FINER GRID IS $0.42 \%$.

Tab. 1. The convergence has been obtained with the angle of attack $\alpha=3^{\circ}$ where the theoretical lift coefficient is $C_{L}=0.3289$. Two types of grids have been compared to determine the effect of chordwise discretizations. As expected, the better description of the leading and trailing edge flow gradients with the biharmonic distribution yields a quicker convergence.

The evolution of the lift coefficient with the angle of attack is shown by the red line in Fig. 4. The lift coefficient predicted by VLM follows almost perfectly the analytical result.

The validation also includes the extraction of the pressure coefficients, in comparison to the analytical solution plotted from equations (10) and (11).

$$
\begin{aligned}
& u=\frac{U_{\infty}}{\cos (\eta / 2)} \cos \left(\frac{2 \alpha-\eta}{2}\right) \\
& c_{p}=\frac{P_{s}-P_{\infty}}{1 / 2 \rho U_{\infty}^{2}}=1-\left(\frac{u}{U_{\infty}}\right)^{2}
\end{aligned}
$$




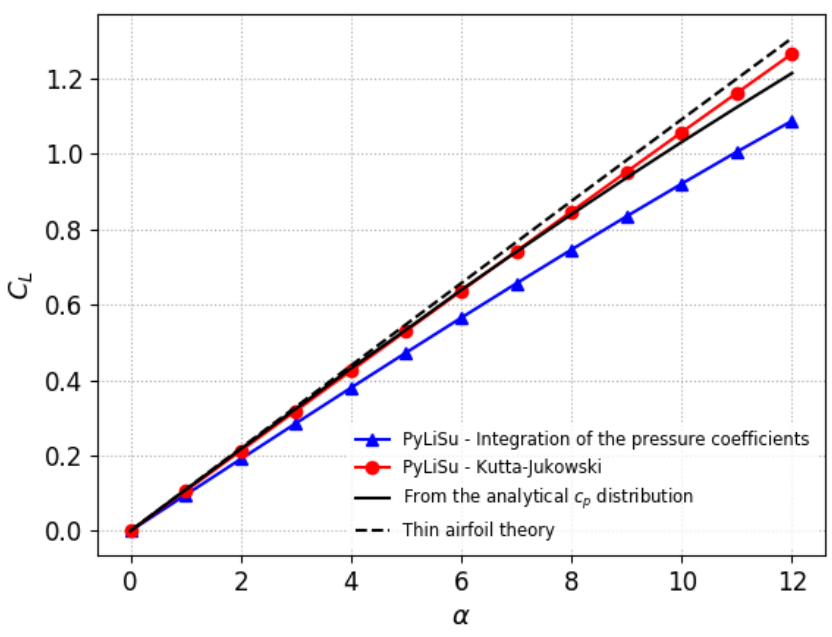

FIGURE 4: LIFT COEFFICIENT AS A FUNCTION OF THE ANGLE OF ATTACK FOR THE FLAT PLATE, PREDICTED BY VLM WITH AR $=1000$ AND COMPARED TO THE 2D ANALYTICAL SOLUTION.

with $\eta$ defined as

$$
\begin{aligned}
& x=c / 2 \cos \eta \\
& y=c / 2 \sin \eta
\end{aligned}
$$

The blue line in Fig. 4 shows the evolution of the lift coefficient with the angle of attack, integrated from the pressure coefficient. This approach underestimates the lift coefficient. To explain this discrepancy, the pressure distribution computed with PyLiSuite is compared to the analytical distribution in Fig. 5. At the leading edge, which corresponds to $\eta=\pi$, the analytical pressure distribution (11) presents a singularity that is not captured by the VLM, hence the discrepancy in lift prediction.

Note that the velocity distribution with PyLiSuite has been calculated using Biot-Savart's law once the circulation, unknown of the problem, was obtained. The extraction of $c_{p}$ has been made in two planes at a distance $\varepsilon$ from the flat plate. Different values of $\varepsilon$ have been tested: an extraction too close to the plate is not accurate and an extraction too far can no longer capture the presence of the plate and its potential effects. In Fig. 5, the extraction from VLM at a distance $\varepsilon=c / 50$ on each side of the plate is compared with the analytical distribution. It fits very well from $x / c=0.1$ to the trailing edge.

NACA0012 airfoil The NACA0012 airfoil is the one tested on the tip-flow experiment. The $2 \mathrm{D}$ validation is based

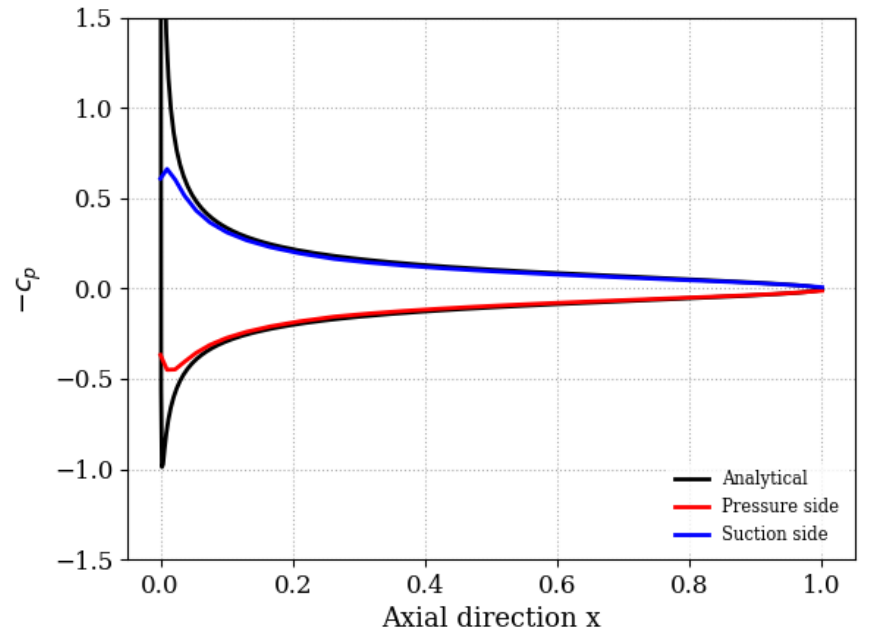

FIGURE 5: PRESSURE COEFFICIENT DISTRIBUTION EXTRACTED FROM VLM COMPARED TO THE 2D ANALYTICAL SOLUTION WITH $\alpha=3^{\circ}$.

\begin{tabular}{c|c} 
Grid size & $C_{L} / C_{L, \mathrm{XFOIL}}$ \\
\hline $10 \times 10$ & 0.9583 \\
$20 \times 20$ & 0.9812 \\
$30 \times 30$ & 0.9868 \\
$40 \times 40$ & 0.9890
\end{tabular}

TABLE 2: CONVERGENCE OF THE LIFT COEFFICIENT OBTAINED WITH PyLiSuite FOR THE NACA0012 AIRFOIL WITH $A R=1000$ AND $\alpha=3^{\circ}$.

on the same parameters as for the flat plate in the previous subsection.

A biharmonic grid is used spanwise and chordwise. The discretization needs to be more refined than for the flat plate probably due to the curvature of the airfoil, especially at the leading edge. Nevertheless, the variations on the lift coefficient are moderate and the $30 \times 30$ grid seems to give a sufficiently accurate evolution of the lift coefficient with the angle of attack, as shown in Table 2. The computation time for this grid is about 1 minute with a regular processor. It increases rapidly to 1 hour when a $100 \times 100$ grid is treated.

The $C_{L}$ values are plotted in Fig. 6. The results from PyLiSuite fit very well the inviscid results obtained with XFOIL [4] for the 2D NACA0012 airfoil.

These two cases validate the approach for high aspect ratios. Three-dimensional cases now have to be treated to complete the validation of the solver before introducing a tip gap in the problem. 


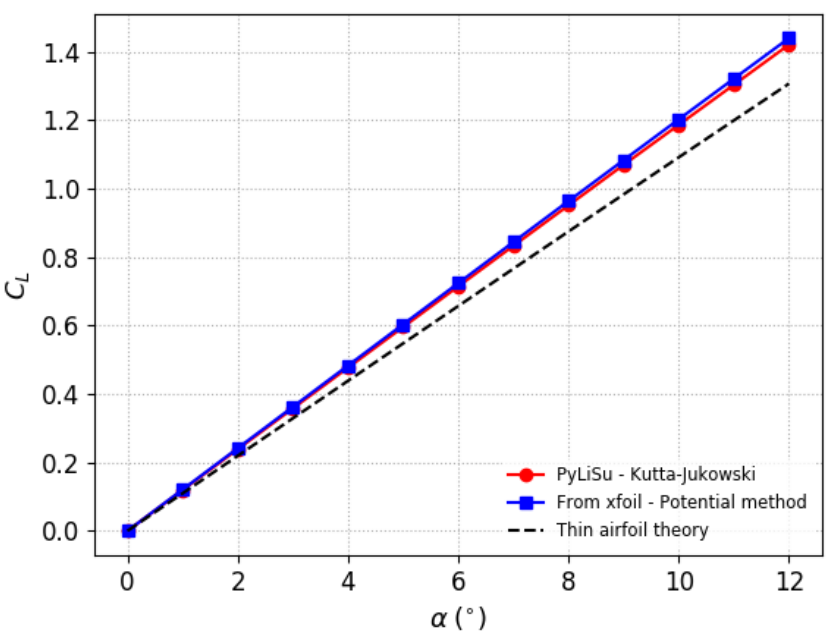

FIGURE 6: LIFT COEFFICIENT AS A FUNCTION OF THE ANGLE OF ATTACK, PREDICTED BY VLM AND COMPARED TO 2D POTENTIAL FLOW PREDICTIONS FROM XFOIL AND THIN AIRFOIL THEORY $C_{L}=2 \pi \alpha$, FOR A NACA0012 AIRFOIL.

\subsection{D validation}

PyLiSuite has been validated on 2D cases by considering very large aspect ratios for the geometries used. Since the vortex lattice method allows going beyond the limitations of lifting line theory, it is also important to validate it by considering threedimensional effects for aerodynamic objects. Moreover, the airfoil considered in the tip-flow experiment presented in section 3.3.1 has a low aspect ratio $A R=2.8$.

Validation on the parabolic wing Another classical case is the parabolic flat wing for which the chord is not constant along the span and varies as follows

$$
c(y)=c_{0} \frac{1-\left(\frac{2 y}{b}\right)^{2}}{1-2 \frac{y c_{0}}{b^{2}} \ln \left(\frac{1-2 y / b}{1+2 y / b}\right)}
$$

Figure 7 shows the grid used for this configuration. The validation is based on the spanwise distribution of the circulation. An analytical expression of the circulation derived from the lifting line theory (large AR) can be obtained:

$$
\Gamma_{p a r}(y)=\frac{\pi b U_{\infty} \alpha}{\left(b / c_{0}+2\right)(1-2 y / b)^{2}}
$$

Figure 8 shows a very good fit of the VLM to the analytical evolution, which enables to validate the module considering 3D effects on this case at high aspect ratio.

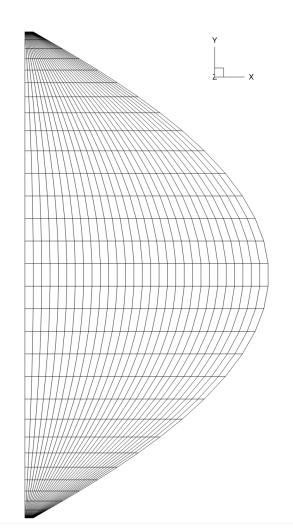

FIGURE 7: GRID USED FOR THE PARABOLIC WING.

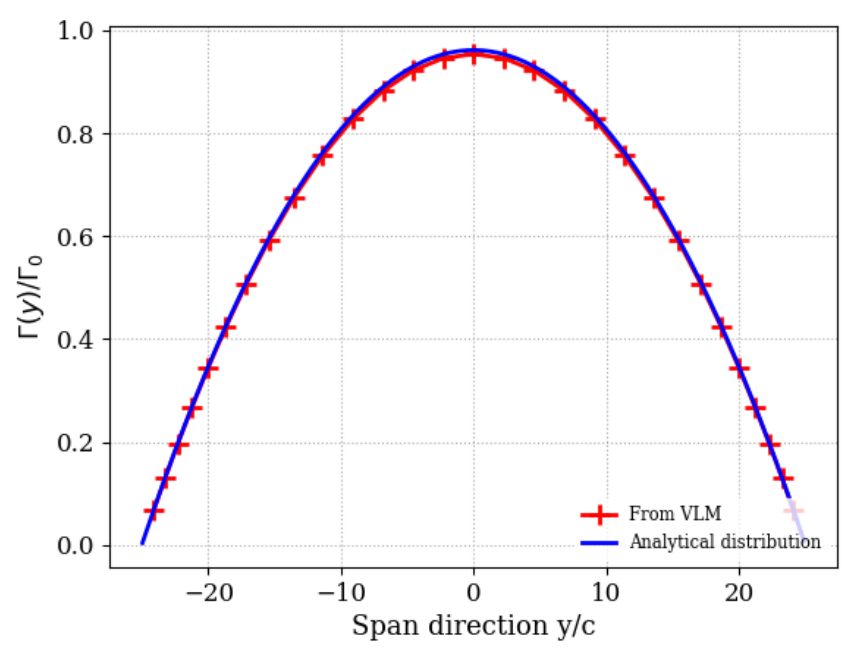

FIGURE 8: CIRCULATION DISTRIBUTION FOR THE PARABOLIC WING WITH AN ASPECT RATIO $A R=75$.

Aspect ratio effect on the flat plate The effect of the aspect ratio on the evolution of the lift coefficient has been investigated by Tuck [22] with a method similar to the VLM. The grid used follows the same distribution as the one described for the $2 \mathrm{D}$ validation. Figure 9 validates the spanwise evolution of $\Gamma(y) / \Gamma_{0}$ with the aspect ratio $A R=b / c$, with $\Gamma_{0}=\pi \alpha U_{\infty} c$. The 


\begin{tabular}{c|c|c|c|c|c}
\hline$U_{\infty}$ & Mach & $P_{\infty}$ & $\alpha$ & $c$ & $R e$ \\
\hline $40 \mathrm{~m} / \mathrm{s}$ & 0.1 & $101325 \mathrm{~Pa}$ & $10^{\circ}$ & $0.2 \mathrm{~m}$ & 550000 \\
\hline
\end{tabular}

TABLE 3: MAIN PARAMETERS OF THE EXPERIMENT.

evolution of the normalized circulation is exactly the same as the one got by Tuck in his article with VLM.

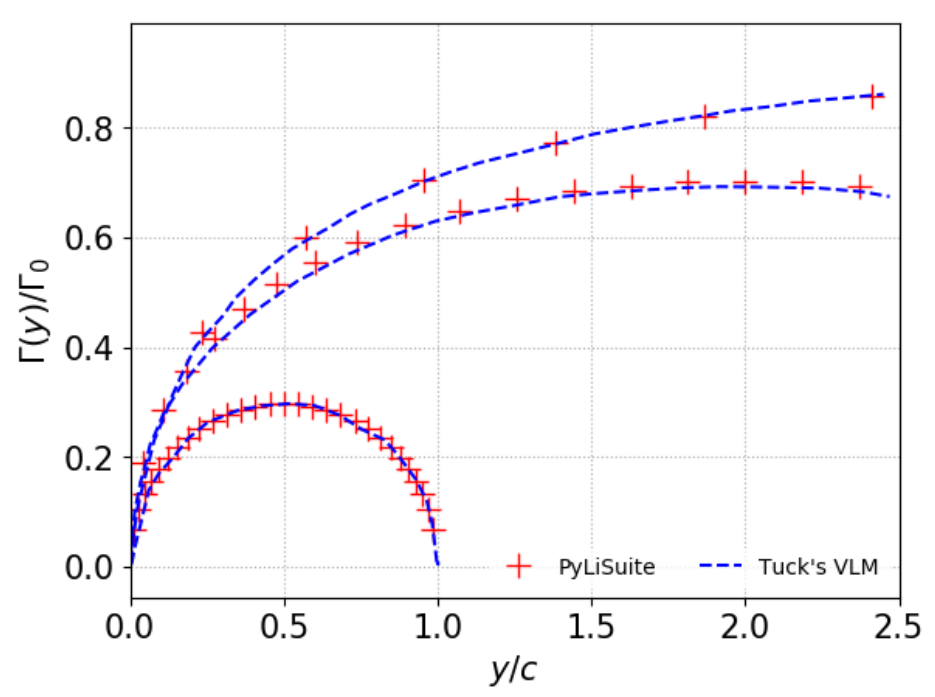

FIGURE 9: SPANWISE DISTRIBUTIONS OF CIRCULATION FOR DIFFERENT ASPECT RATIOS PREDICTED BY PyLiSuite COMPARED TO TUCK'S VLM.

\subsection{Evaluation on tip-leakage flow}

3.3.1 Presentation of the rig An experimental parametric study on the effects of the tip gap size on the flow has been performed in the S2l subsonic wind tunnel, located at the ONERA Meudon center. The flow is investigated around a NACA0012 airfoil whose distance from its tip to the endwall can be adjusted. The size ranges from sizes representative of actual compressors $(1-2 \%$ chord length) to larger tip gaps (13\% chord length), where viscous effects have much less impact. Figure 10 shows a representation of the experimental setup where the tip gap size $\tau$ can be varied. Table 3 summarizes the main parameters of the experiment. The value of the angle of attack $\alpha$ was chosen to have a sufficient blade loading without getting too close to flow separation. Five-hole probe measurements were performed in a plane situated downstream of the blade for a constant axial position $x=1.05 c$. The results in that plane will be used for the comparisons with the VLM module PyLiSuite presented in the present paper.

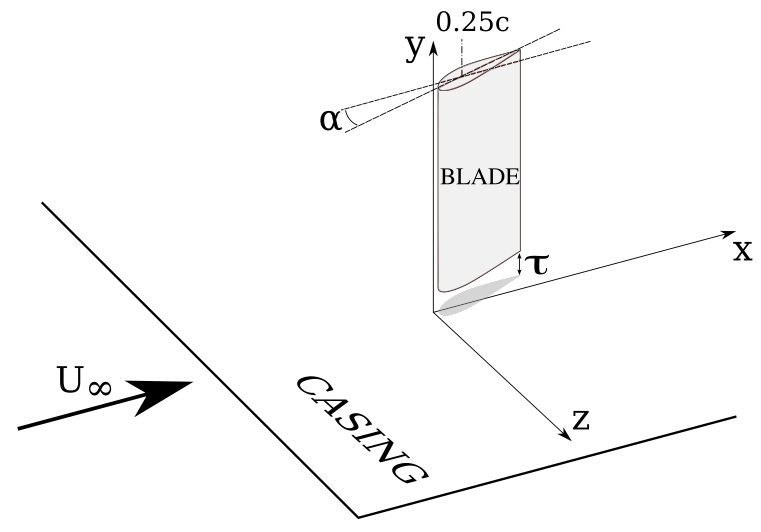

FIGURE 10: REPRESENTATION OF THE S2l SETUP USED FOR THE COMPARISONS.

Up to this point, the VLM has only been used without considering endwalls near the tip of the surfaces discretized, i.e. with an infinite tip clearance. The purpose of this paper is to compare the results brought by this approach with the experiment regarding tip-leakage flow. The formation of the vortex and how it develops depending on the size of the gap is of particular interest. This section shows how TLV is predicted by PyLiSuite without the presence of the endwall first and how it is modified when the endwall is added.

3.3.2 Flow with an infinite tip clearance Before showing the results from PyLiSuite with a tip clearance, it is interesting to consider a configuration without a tip clearance, which can also be seen as a configuration with an infinite tip gap size. Figure 11 shows how the tip vortex is predicted. As explained in section 2, it results from the superposition of the horseshoe vortices attached to the panels on both sides of the blade. Each side of the blade is here discretized into $40 \times 40$ panels. The roundness of the predicted vortex is remarkable.

3.3.3 Flow in presence of the tip clearance Let us now add the presence of a casing. Two conditions of symmetry are here applied to replicate the presence of the endwalls:

1. At the root of the blade, to ensure that the shed vorticity tends towards zero, corresponding to the presence of the endwall with no clearance (hub)

2. At the tip of the blade by placing another blade, $2 b$ long, accounting for the first condition of symmetry, at a distance $2 \tau$ from the other blade, exactly as Lakshminarayana and Horlock proceeded with their slotted wing. 


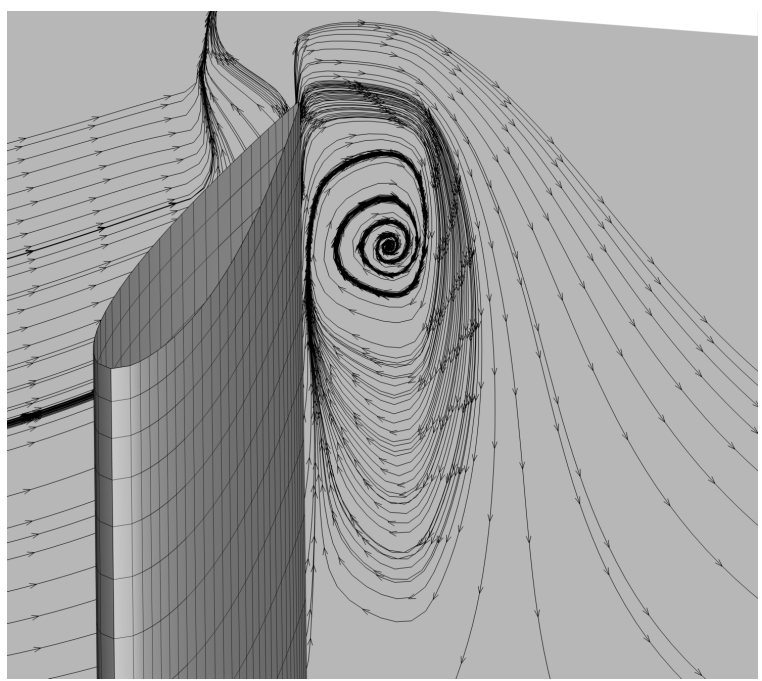

FIGURE 11: STREAMLINES IN A PLANE DOWNSTREAM OF THE BLADE PERPENDICULAR TO THE INFLOW AT $\mathrm{x}=1.05 \mathrm{c}$, COMPUTED WITH VLM.

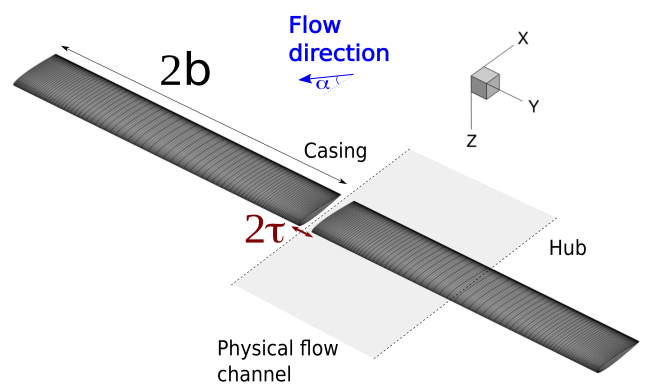

FIGURE 12: GRID USED WITH VLM WITH THE CONDITIONS OF SYMMETRY WITH $200 \times 200$ NUMBER OF POINTS.

The configuration considered is shown in Fig. 12.

\section{Topology of the flow}

Figures 13 and 14 compare the pitchwise component of the velocity in the measurement plane downstream of the blade for the higher gap ratio $\tau / c=13 \%$. The results are first compared for this gap size, which is the largest of the different sizes tested. The comparison is more appropriate for this gap size since po- tential effects are predominant. Viscous effects, especially due to the presence of the endwall boundary layer, would have to be taken into account for smaller gap sizes.

The TLV is predicted by VLM even though the streamlines are deformed in the wake of the blade. This is due to the influence of the legs of the horseshoe vortices shed downstream of the blade with VLM. Indeed, a singularity exists at the center of the horseshoe vortices. Regarding the intensity, the VLM predicts comparable values, slightly higher, with more intense gradients in the core of the TLV. The position of the vortex core is also different: in the experiment, pitchwise, the vortex core is farther from the blade $\left(z_{T L V}-z_{T E} \approx 0.1\right)$. The point of detachment is represented by the VLM and the trajectory of the TLV is only determined by the distribution of the horseshoe vortices, aligned with the $x$ axis (here, $z_{T L V}-z_{T E} \approx 0.005$ ).

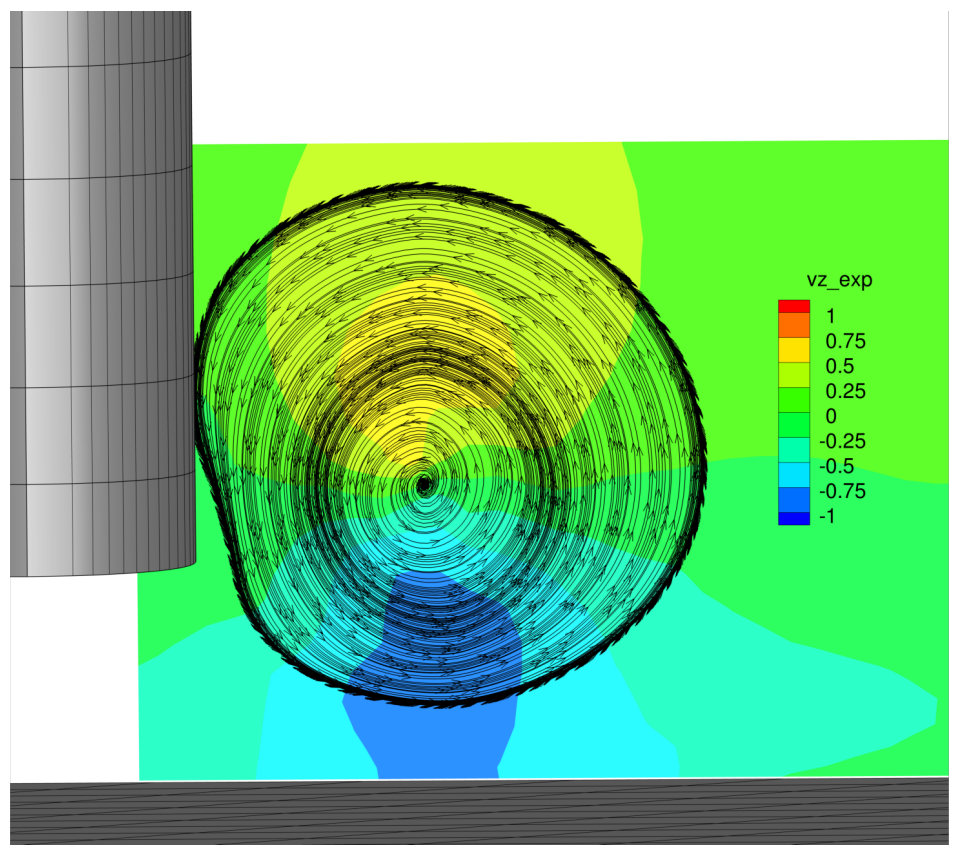

FIGURE 13: PITCHWISE COMPONENT OF THE VELOCITY MEASURED IN THE PLANE OF REFERENCE OF THE EXPERIMENT.

Figures 15 and 16 show the static pressure coefficient in the same plane from the experimental measurements and PyLiSuite respectively, for the same tip gap size $\tau=13 \% c$. The TLV predicted by the VLM has a comparable size to the one obtained experimentally.

The pressure deficit observed with the VLM is however more important. The maximal depression below -1 for the pressure coefficient is spread over a wider area with the VLM than with the experiment. Moreover the depression predicted is more 


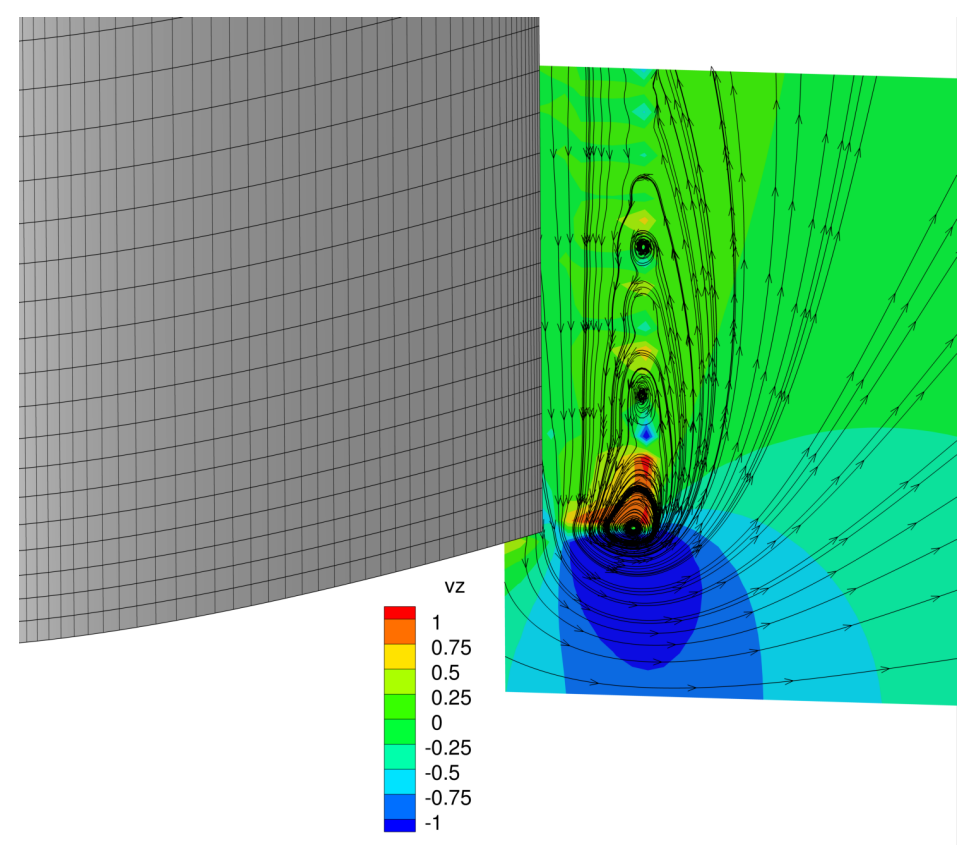

FIGURE 14: PITCHWISE COMPONENT OF THE VELOCITY COMPUTED WITH VLM IN THE PLANE OF REFERENCE.

intense with PyLiSuite. Several explanations can be put forward:

1. The absence of viscous effects modifies the vena contracta in the clearance. The effective cross section corresponds to the geometrical gap, without reduction by the viscous effects.

2. Another difference is the position of the TLV relative to the static pressure minimum. In the VLM, the position of the TLV is controlled by the distribution of the horseshoe vortices and their intensities.

3. Viscous losses are not accounted for with the VLM, this is a purely potential method. In particular, no total pressure losses are considered.

\section{Sensitivity to the gap size}

Finally, the sensitivity to the size of the gap is investigated. Figure 17 shows the distributions of bound circulation for three gap sizes. As expected, the bound circulation is the lowest for the largest gap. In other words, the configuration with the largest tip gap generates less lift due to the larger induced drag associated to the more intense TLV. This can be seen through the slope of the circulation near casing $(y / c=0)$ which is higher as the gap is enlarged. This corresponds to a larger vorticity shed downstream, which is indeed related to $\frac{d \Gamma}{d y}$. In terms of tip loading, the larger the gap, the lower the loading with this approach, referring to equation (2).

Table 4 contains the values of $D_{\text {design }}$ as defined in section

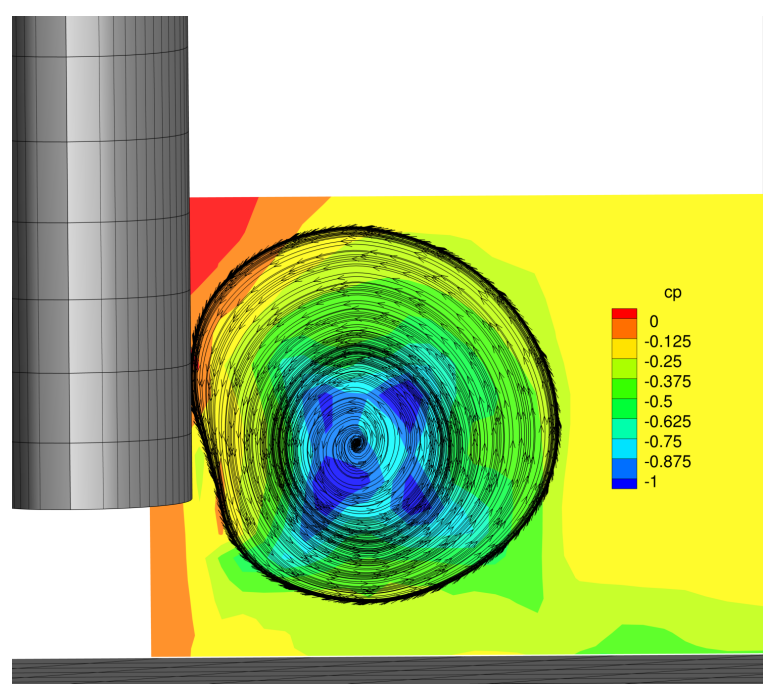

FIGURE 15: PRESSURE COEFFICIENT FIELD MEASURED IN THE EXPERIMENT, WITH STREAMLINES, $\tau / c=13 \%$.

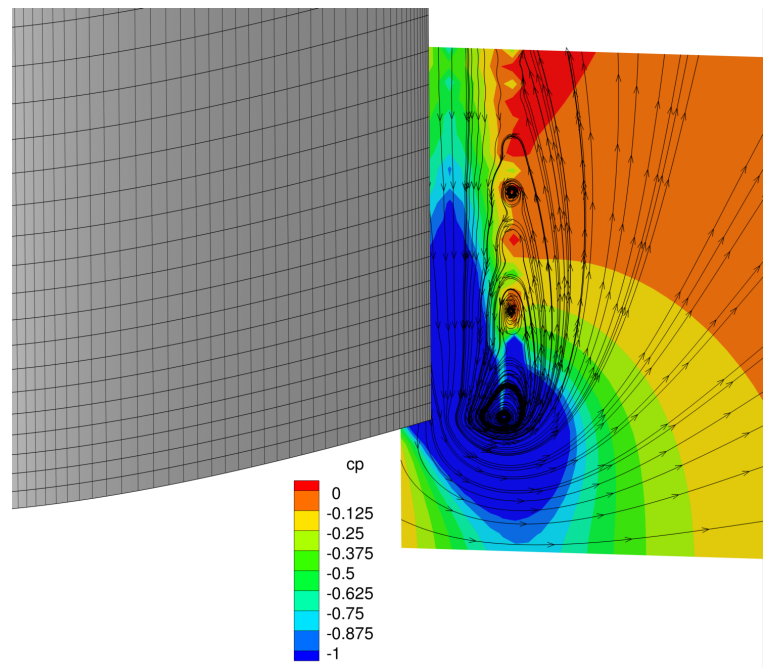

FIGURE 16: PRESSURE COEFFICIENT FIELD COMPUTED FROM PyLiSuite WITH STREAMLINES FOR $\tau / c=13 \%$.

2.2. Consistently with the distributions observed, the criterion shows that the largest tip gap size deviates more from the equivalent constant work design.

\begin{tabular}{|l|c|c|c|}
\hline$\tau / c(\%)$ & 2.5 & 6 & 13 \\
\hline$D_{\text {design }}$ & 0.066 & 0.097 & 0.122 \\
\hline
\end{tabular}

TABLE 4: VALUES OF $D_{\text {Design }}$ FOR THE THREE TIP GAP SIZES CONSIDERED. 


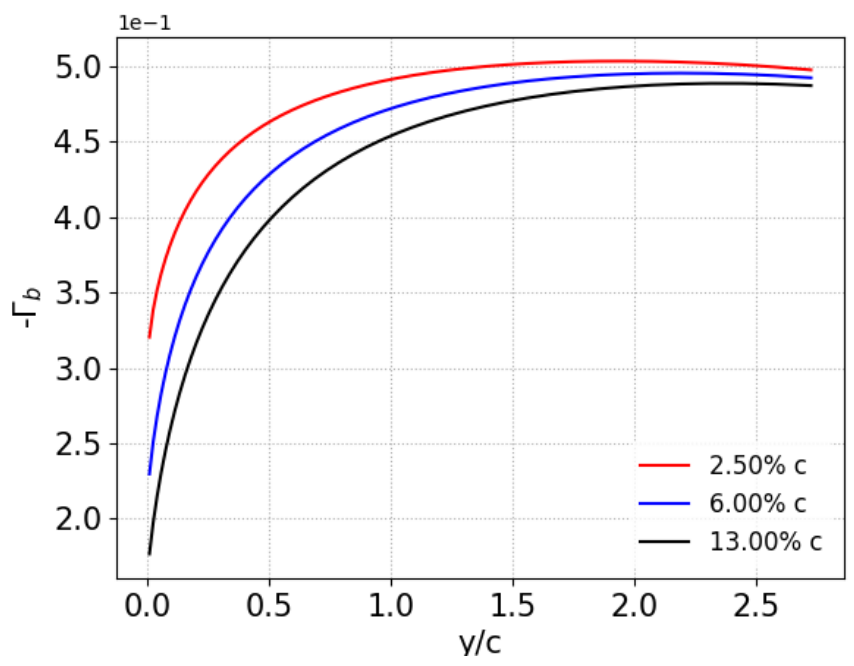

FIGURE 17: SPANWISE DISTRIBUTION OF THE BOUND CIRCULATION PREDICTED BY VLM FOR THREE GAP SIZES.

Another way to see the influence of the TLV circulation is to have a look at the outlet angle distribution $\alpha_{e}$ calculated from the velocity components in the measurement plane obtained with Biot-Savart's law. Figure 18 shows the distributions for the same tip gap sizes as in Fig. 17. With an increasing tip gap size, the flow is less deviated near the tip corresponding indeed to a lower value for the circulation, i.e. the blade loading.

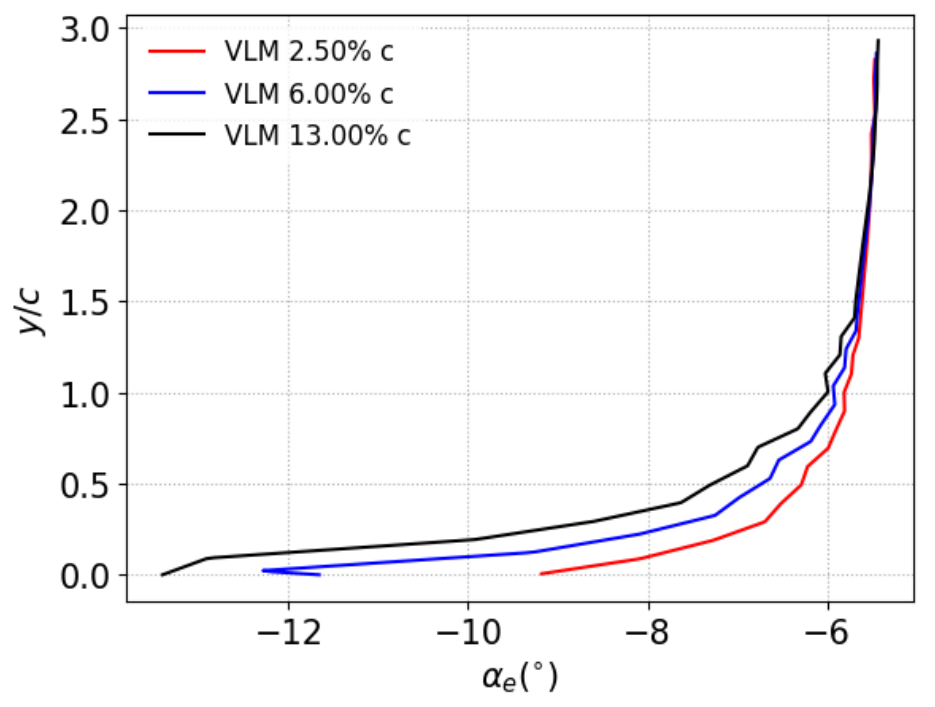

FIGURE 18: SPANWISE DISTRIBUTION OF THE OUTLET ANGLE.
Figure 19 shows the evolution of the TLV circulation with the gap size, for the experiment and the simulation. The total circulation has been computed:

1. In the experiment, by integration of the axial vorticity in the measurement plane

2. In PyLiSuite, by integration of the spanwise distribution of $\frac{d \Gamma}{d y}$.

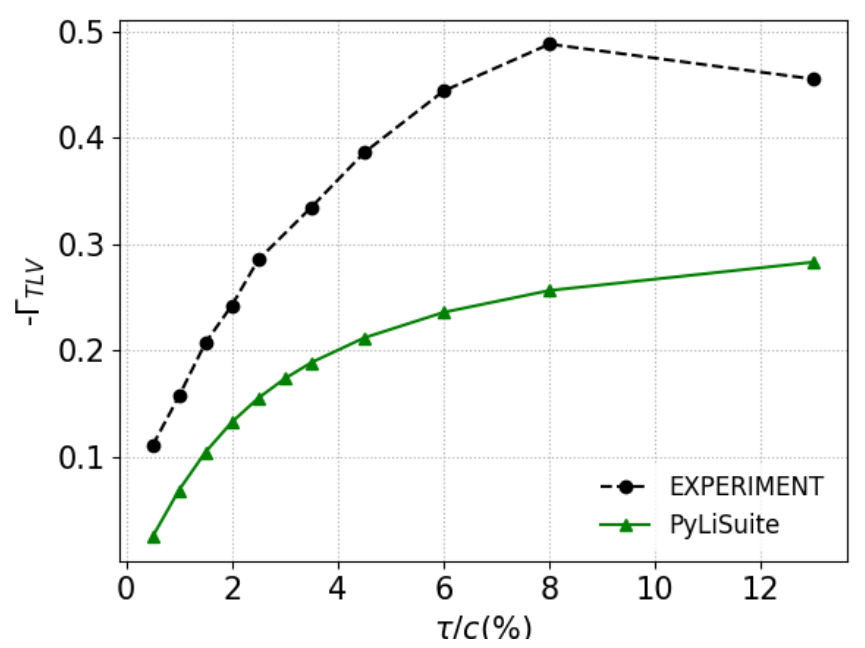

FIGURE 19: TLV CIRCULATION AS A FUNCTION OF THE TIP GAP SIZE. COMPARISON BETWEEN EXPERIMENTAL AND NUMERICAL RESULTS.

The circulation of the TLV is underestimated by the VLM but the increase of the TLV circulation with the tip gap size is well captured.

\section{CONCLUSIONS}

The objective of this paper was to validate the module PyLiSuite developed, based on the Vortex Lattice Method (VLM), and use it to assess the capabilities of this approach to predict TLF. The VLM is a potential method which consists in discretizing an object into panels of vorticity, replicating the presence of the object by the existence of elementary circulations.

The validation was divided into two parts:

1. A two-dimensional part, where the basic aerodynamic characteristics were checked in two cases. In the cases of the flat plate and the NACA0012 airfoil, the results given by the solver are validated. 
2. A three-dimensional part where the spanwise effects are accounted for. For the flat plate, the results are satisfying over a wide range of aspect ratios. For the parabolic wing, the results are good in comparison with the analytical results derived from lifting line theory, valid for high aspect ratio.

The results of the module were then compared with the experimental measurements performed in a wind tunnel on a NACA0012 airfoil with adjustable tip gaps. The comparison has given interesting results regarding the formation of the tip leakage vortex (TLV). The TLV is described by the VLM. For a large clearance, the size of the vortex is well predicted, but the pressure deficit is overestimated. When the gap size is reduced, the decrease of the TLV circulation is captured. The solver still needs to be improved, mostly regarding the influence of viscosity, not considered in that kind of approach. Another improvement concerns the computation time which could allow finer discretizations to be treated much more quickly. A solution could be to replace the symmetry conditions with two plates replicating the presence of the endwalls at casing and hub, and to apply a slip condition on them. Further investigation is needed regarding the extension of the plates and their discretization. This module aims at being used for parametric studies on TLF on more complex configurations in a reasonable computation time (i.e. about one minute).

\section{REFERENCES}

[1] John David Anderson Jr. Fundamentals of aerodynamics. Tata McGraw-Hill Education, 2010.

[2] Vincent Brion. Stabilité des paires de tourbillons contrarotatifs : application au tourbillon de jeu dans les turbomachines. PhD Thesis, Ecole polytechnique, 2009.

[3] J.D. Denton. Loss Mechanisms in Turbomachines. Technical report, The American Society of Mechanical Engineers, New York, 1993.

[4] Mark Drela. Xfoil: An analysis and design system for low reynolds number airfoils. In Low Reynolds number aerodynamics, pages 1-12. Springer, 1989.

[5] Edward M. Greitzer, Choon Sooi Tan, and Martin B. Graf. Internal flow: concepts and applications, volume 3. Cambridge University Press, 2007.

[6] Donald A. Hoying, Choon S. Tan, Huu Duc Vo, and Edward M. Greitzer. Role of blade passage flow structures in axial compressor rotating stall inception. In ASME 1998 International Gas Turbine and Aeroengine Congress and Exhibition, pages V001T01A138-V001T01A138. American Society of Mechanical Engineers, 1998.

[7] Richard M. James. On the remarkable accuracy of the vortex lattice method. Computer Methods in Applied Mechanics and Engineering, 1(1):59-79, 1972.
[8] Joseph Katz and Allen Plotkin. Low-Speed Aerodynamics. 2001.

[9] S. A. Khalid, A. S. Khalsa, I. A. Waitz, C. S. Tan, E. M. Greitzer, N. A. Cumpsty, J. J. Adamczyk, and F. E. Marble. Endwall Blockage in Axial Compressors. Journal of Turbomachinery, 121:499-509, 1999.

[10] C. C. Koch. Stalling pressure rise capability of axial flow compressor stages. Journal of Engineering for Power, 103(4):645-656, 1981.

[11] C. C. Koch and L. H. Smith. Loss sources and magnitudes in axial-flow compressors. Journal of Engineering for Power, 98(3):411-424, 1976.

[12] B. Lakshminarayana. Methods of predicting the tip clearance effects in axial flow turbomachinery. 1970.

[13] B. Lakshminarayana and J.H. Horlock. Tip-clearance flow and losses for an isolated compressor blade. Technical report, Aeronautical Research Council Reports and Memoranda, London, 1963.

[14] B. Lakshminarayana and JH t Horlock. Leakage and secondary flows in compressor cascades. HM Stationery Office, 1965.

[15] Dean T. Mook and Ali H. Nayfeh. Application of the Vortex-Lattice Method to High-Angle-of-Attack Subsonic Aerodynamics. SAE Transactions, 94:517-532, 1985.

[16] I. K. Nikolos, D. I. Douvikas, and K. Papailiou. A Method for the Calculation of the Tip Clearance Flow Effects in Axial Flow Compressors. The American Society of Mechanical Engineers, pages 1-13.

[17] Olufemi Okurounmu and James McCune. Threedimensional vortex theory for axial compressor blade rows at subsonic and transonic speeds. AIAA Journal, 8(7):12751282, 1970.

[18] Olufemi Okurounmu and James McCune. Lifting Surface Theory of Axial Compressor Blade Rows: Part I-Subsonic Compressor. AIAA Journal, 12(10):1363-1372, 1974.

[19] Ludwig Prandtl. Tragflügeltheorie. Technical report, Göttingen Universität, Göttingen, February 1919.

[20] Dan A. Rains. Tip clearance Flows in Axial Compressors and Pumps. PhD Thesis, California Institute of Technology, Pasadena, California, 1954.

[21] J.A. Storer and N.A. Cumpsty. An Approximate analysis and prediction method for tip clearance loss in axial compressors. The American Society of Mechanical Engineers, (93-GT-140):1-10, 1993.

[22] E. O. Tuck. Some accurate solutions of the lifting surface integral equation. The ANZIAM Journal, 35(2):127-144, 1993.

[23] Huu Duc Vo, Choon S. Tan, and Edward M. Greitzer. Criteria for spike initiated rotating stall. Journal of turbomachinery, 130(1):011023, 2008.

[24] Donghyun You, Meng Wang, Parviz Moin, and Rajat Mittal. Large-eddy simulation analysis of mechanisms for vis- 
cous losses in a turbomachinery tip-clearance flow. Journal of Fluid Mechanics, 586:177-204, April 2007. 